\title{
Free operant single and double alternation in the albino rat: A demonstration
}

\author{
MARY NELL TRAVIS-NEIDEFFER and JERRY D. NEIDEFFER \\ Department of Behavioral Sciences, Kansas Wesleyan, Salina, Kansas 67401
}

and

\author{
STEPHEN F. DAVIS \\ Emporia State University, Emporia, Kansas 66801
}

\begin{abstract}
This experiment assessed the ability of rats to learn uncued free operant single- and double-alternation tasks. Each of six male albino rats was trained to single and double alternate responses between two levers in an operant conditioning chamber. All rats quickly mastered the single-alternation task. Accuracy improved with increased double-alternation training and was above the level predicted by chance. However, double-alternation accuracy was higher when extra responses were allowed (e.g., LLLRR was reinforced as was LLRR). The rats in this experiment were totally unable to perform two correct double alternations in sequence (i.e., LLRRLLRR or RRLLRRLL).
\end{abstract}

The now classic experiments of $\mathrm{W}$. S. Hunter have been cited as support for the notion that rats appear to be unable to successfully execute responses in a doublealternation sequence. More specifically, Hunter (1920), using a "temporal maze," found that rats were limited in their ability to learn to alternate sequences of two left turns with two right turns. Similarly, the results of more recent research using a straight alley (Bloom \& Capaldi, 1961; Ludvigson \& Sytsma, 1967) and a modified WGTA apparatus (Livesey, 1965) have supported Hunter's (1920) conclusion that rats are unable to double alternate responses unless specific external stimuli that are uniquely correlated with the next correct response are provided.

On the other hand, other studies reporting the development of double-alternation performance have appeared in the literature. For example, Schlosberg and Katz (1943) attempted to establish free operant true double alternation, using a single-lever operant conditioning chamber. They defined true double alternation as "the ability to complete the RRLL sequence without external differentiating stimulus cues to guide the behavior at the choice point" (Schlosberg \& Katz, 1943). Rats were required to push the lever twice to the right and then twice to the left in order to obtain reinforcement. Although the animals could insert extra responses into the sequence and continue to be rein-

The authors sincerely thank Stephen Winokor for his advice and assistance throughout this project. Additionally, the authors extend sincere appreciation to J. W. Voorhees and Patty Randolph for their assistance in collection of the data. Requests for reprints should be addressed to Mary Nell Travis-Neideffer, Department of Behavioral Sciences, Kansas Wesleyan, Salina, Kansas 67401. forced (e.g., RRLL would be reinforced, as would RRRLL), Schlosberg and Katz reported the occurrence of some errorless double-alternation sequences (i.e., no extra responses in the RRLL sequence). In fact, one rat reportedly produced an errorless series of 35 consecutive double alternations. Despite their apparent success, the Schlosberg and Katz (1943) data have been criticized (Kelleher, 1966) for the presence of a possible confounder. Namely, each correct response in the doublealternation sequence produced a distinctive click, whereas incorrect responses produced no such stimulus. These clicks could have served as conditioned reinforcers that selectively strengthened correct responses.

More recently, Heise, Keller, Khavari, and Laughlin (1969) showed that rats could learn an uncued doublealternation "go/no-go" schedule in an operant conditioning chamber. Likewise, Khavari (1970) required rats to press one of two bars that were inserted into an operant conditioning apparatus on each trial. The correct bar for each trial was determined by a double-alternation sequence. If a rat made an incorrect response, both bars were withdrawn, no reinforcement occurred, and the trial was terminated. Under these conditions, rats significantly increased the proportion of correct responses over 1,500 trials but were still significantly inferior to subjects receiving visual cues during training.

As with the Schlosberg and Katz (1943) study, procedural aspects of the Heise et al. (1969) and Khavari (1970) studies have been examined. It would appear that these studies also cannot yield an unequivocal answer regarding the rat's ability to double alternate responses without the benefit of exteroceptive cues. Realistically, Heise et al. (1969) examined whether rats could learn an alternating reinforcement schedule, and not if the sub- 
jects could learn to double alternate responses for reinforcement. Even Khavari (1970), who provided perhaps the clearest evidence for double-alternation responding, pointed out that the task was extremely difficult and, as noted above, that rats given visual cues for responding showed superior learning. Furthermore, Khavari (1970) reinforced each correct response rather than each correct double alternation. Certainly, it would be of interest to know if rats can learn to double alternate when each correct alternation, rather than each correct response, is reinforced.

The present experiment attempts to provide an unequivocal test of the rats' ability to double alternate responses in a free operant task when only each correct double-alternation sequence is reinforced. Additionally, efforts were made to assess the relative ease or difficulty with which single- and double-alternation sequences are learned and to examine the effects of a fixed-ratio (FR 2) schedule of reinforcement for double-alternation responding. Hunter and Nagge (1931) noted that exceptional rats that eventually mastered the LLRR problem in the "temporal maze" were unable to learn the series LLRRLL or LLRRLLRR (i.e., an FR 2 schedule of double alternation). Ferster (1960), however, found that pigeons performed more accurately on a matching-tosample task when they were reinforced on a small FR schedule than when they were reinforced for every correct match (CRF). Similarly, Ferster (1958) found that a chimpanzee's performance on a counting schedule (i.e., the chimpanzee was required to press exactly three times for food reinforcement) was more accurate when reinforced on an FR 33 schedule as opposed to a CRF schedule. If complex behaviors such as these are improved when responses are reinforced on ratio schedules, then it would be of interest to know if a rat's accuracy on a free operant double-alternation task would be similarly improved by reinforcing successful double alternations on a small ratio schedule.

\section{METHOD}

\section{Subjects}

The subjects were six 150-day-old male rats from the Holtzman Company, Madison, Wisconsin. Although these animals had previously served in another experiment, they were naive with respect to the procedures of the present experiment. The rats were individually housed and maintained under conditions of constant illumination. Water was freely available. Each rat was reduced to $85 \%$ of his free-feeding weight prior to commencement of the study and maintained at this weight by controlled feedings of Purina Rat Chow pellets.

\section{Apparatus}

The experimental apparatus consisted of three GrasonStadler (Model E3125A-100) operant conditioning chambers. Each chamber was housed in a sound-attenuating chest and was equipped with two Ralph Gerbrands (Model G6312) rat levers, each mounted $9 \mathrm{~cm}$ from the grid floor. The levers were mounted on the back wall of the chamber, opposite to the food tray, and were themselves separated by $10 \mathrm{~cm}$. A clean, absorbent cage liner was placed below the grid floor of each chamber and replaced after each rat's experimental session. Relay program- ming and recording equipment of the type described by Ferster and Skinner (1957) controlled the experiment and recorded the data.

\section{Procedure}

Preliminary training. Each of the six rats was permanently assigned to one of the three testing chambers. Assignment was random, with the restriction that two rats were assigned to each chamber.

Preliminary training for each rat began with two 1-h adaptation sessions. During the adaptation sessions, the food magazine was inoperative and the levers were absent. Following adaptation, all rats were exposed to 2 days of lever-absent magazine training. On these days, each rat was presented $6045-\mathrm{mg}$ food pellets (P. J. Noyes, Formula A) on a variable-time (VT) 1-min schedule. All rats were observed to reliably eat from the food tray by the end of magazine training.

CRF training. On Days 1 and 2 of CRF training, only one bar was present. For half of the animals, leverpress training began on the right bar, and the other rats began with the left bar. Day 2 of this training involved removing the bar that had been present on Day 1 and inserting the other bar. Both levers were present in the chambers on Days 3 and 4 . Each animal remained in the apparatus until 150 successful barpresses had been completed on each of these 4 days. Each successful barpress was followed by one 45-mg food pellet. There were no restrictions on distribution of responses between the two levers on Days 3 and 4 .

Single-alternation training (Days 1-24). Immediately following CRF training, all animals were required to single alternate responses for reinforcement. No exteroceptive cues provided information concerning the correctness or incorrectness of responses. Two rats ( 1 and 4 ) were trained to alternate from the left bar to the right bar, and the remaining four rats $(2,3,5$, and 6) were trained to alternate from the right bar to the left bar. A 45-mg food pellet was delivered after each correct alternation. For example, if the task was to alternate from right to left, the rat was reinforced only if one response on the right bar was followed by one response on the left bar. If a rat made more than one response before alternating, or if responding began on the wrong bar, the programming equipment automatically reset and made delivery of a reinforcer impossible until a correct sequence occurred. Sessions were $45 \mathrm{~min}$ in length.

Double-alternation training (Days 25-69). Immediately following single-alternation training, all animals were shifted to the double-alternation task. During this phase, Rats 1 and 4 were required to make two responses on the left lever followed by two responses on the right lever and the remaining animals were required to make two responses on the right lever followed by two responses on the left lever. A single food pellet was delivered upon the completion of a correct sequence. As was the case during single-alternation training, no exteroceptive cues were provided concerning the correctness of responses. However, no penalty was assessed for making more than two responses on the first bar before alternating to the second bar. The only requirement was that the rat make at least two responses on the first bar followed by two responses on the second bar. Sessions were $45 \mathrm{~min}$ in length.

FR 2 double-alternation training (Days 70-78). At the end of double-alternation training, all rats were exposed to 9 days of FR 2 double-alternation training. FR 2 training was similar to double-alternation training, with two exceptions. First, all rats were required to produce two correct double-alternation sequences before being reinforced (i.e., LLRRLLRR or RRLLRRLL). Second, due to the nature of the program, foodpellet deliveries would not occur if more than two responses were made on the first bar before moving to the second bar. Session length was $45 \mathrm{~min}$.

Double-alternation retraining (Days 79-96). Upon completion of FR 2 double-alternation retraining, all rats were retrained on the double-alternation schedule described above. Sessions were $45 \mathrm{~min}$ in length. 
True double-alternation training (Days 97-126). At the end of double-alternation retraining, all rats were introduced to a variant of the double-alternation task. The animals were still required to make two responses on the first bar before switching to the second bar. However, a penalty was assessed if the rat made more than two responses on the first bar. If excess responding occurred, the programming equipment automatically reset, disallowing delivery of a reinforcer until a correct sequence occurred. Session length was $45 \mathrm{~min}$.

FR 2 double-alternation retraining (Days 127-131). Subsequently, all animals were reexposed to the FR 2 double-alternation task previously described. Sessions continued to be $45 \mathrm{~min}$ in length.

True double-alternation retraining (Days 132-160). All rats were then retrained on true double alternation. Session length remained $45 \mathrm{~min}$. The experiment terminated with the completion of this phase.

\section{RESULTS}

Figure 1 shows the mean percent correct alternations on all tasks for the six subjects. The ordinate represents the percentage of the total number of response sequences within a day that were correct, and the abscissa displays consecutive days of training. In the case of single alternation, the percentage of correct alternations was derived by dividing the total number of correct two-response sequences (LR or RL) by the total number of two-response sequences. In the case of double alternation and true double alternation, the total number of correct four-response sequences (LLRR or RRLL) was divided by the total number of four-response sequences. For FR 2 double-alternation training, the percentage of correct alternations was determined by dividing the number of correct eight-response sequences (LLRRLLRR or RRLLRRLL) by the total number of eight-response sequences.

Each of the six rats readily acquired single alternation, with no apparent differences between learning the LR sequence (Rats 1 and 4) and learning the RL sequence (Rats 2, 3, 5, and 6). The mean percent correct sequences for the last 3 days of single-alternation training (Days 1 3) was found to be significantly higher than the mean percent correct sequences for the first 3 days (Days 22 24) $[\mathrm{t}(5)=6.93, \mathrm{p}<.001]$, thus verifying that the percentage of correct single alternations increased over the course of this training.

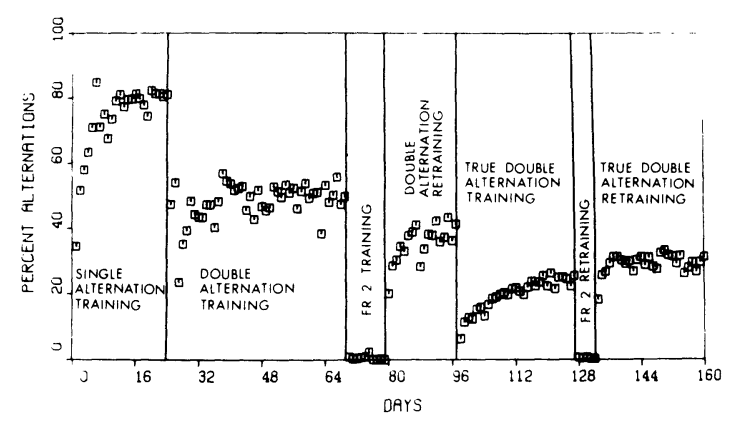

Figure 1. Mean percent correct alternations for Rats 1-6.
Results of double-alternation and true doublealternation training clearly showed that the rats did learn to perform these tasks, although their accuracy was not as great as on the single-alternation task. Comparing the last 3 days of the single-alternation training (Days 2224) with the last 3 days of both double-alternation training (Days 67-69) and true double-alternation training (Days 125-127) confirmed this impression. The mean percent correct sequences was higher for single alternation than for double alternation $[\mathrm{t}(5)=4.26$, $\mathrm{p}<.01]$ or for true double alternation $[\mathrm{t}(5)=12.41$, $\mathrm{p}<.001]$. However, accuracy on each day of both double-alternation tasks, with the exception of the 1 st day of true double-alternation training, was above the $6 \%$ that would be expected by chance. The mean percent correct sequences for the last 3 days of doublealternation training (Days 25-27) was found to be significantly higher than the corresponding mean for the first 3 days (Days 67-69) [ $t(5)=2.19, \mathrm{p}<.05]$, verifying that the subjects significantly improved their performance on the double-alternation task during training. Similarly, Days 98-100 of true double-alternation training were compared with Days 125-127. The mean percent correct sequences for the last 3 days of this training was found to be significantly higher than the mean percent correct sequences for the first 3 days $[t(5)=6.73, p<.001]$, demonstrating that accuracy on this task also improved with training. It may be interesting to note, however, that the true double-alternation task, which did not permit more than two responses on the first lever before switching to the second, did not appear to support the same degree of accuracy as the double-alternation task. Comparing the last 3 days of double-alternation training (Days 67-69) with the last 3 days of true double-alternation training (Days 125-127) substantiated this impression, with the mean percent correct sequences for the double-alternation task exceeding the mean percent correct sequences for the true double-alternation task $[\mathrm{t}(5)=4.55, \mathrm{p}<.01]$.

Although there was evidence of at least some learning of the double-alternation and true double-alternation tasks, there was no indication that any learning of the FR 2 double-alternation task occurred. Each rat's accuracy dropped to almost zero and remained at that level for the 9 days of FR 2 double-alternation training and the 5 days of FR 2 double-alternation retraining. However, double-alternation responding recovered after FR 2 double-alternation training (i.e., double-alternation retraining), as did true double-alternation responding (true double-alternation retraining) after FR 2 doublealternation retraining.

\section{DISCUSSION}

The primary purpose of this experiment was to provide an unequivocal test of the rat's ability to double alternate responses in a free operant task when each correct double-alternation sequence, rather than each correct response, was reinforced and when no experimenter-provided, exteroceptive cues were available. Additionally, an effort was made to assess the relative ease 
with which single- and double-alternation sequences are learned and to determine the effect of an FR 2 schedule of reinforcement on accuracy of responding on a double-alternation task.

The present data clearly showed that rats are capable of some learning of an uncued free operant double-alternation task. Performance was better when more than two responses were permitted on the first bar (double alternation) than when extra responses were not allowed (true double alternation). Nonetheless, accuracy on both double-alternation and true doublealternation tasks was well above the $6 \%$ level that would be expected by chance alone, and accuracy improved over days of training in both cases.

Even though rats were able to perform a true doublealternation task for reinforcement, the single-alternation task generated a higher degree of accuracy than did either of the double-alternation tasks. Hunter (1920) reported that rats easily mastered a single-alternation task in a maze but did not show any significant progress toward mastery of a double-alternation task. The data presented here further support the conclusion that rats are quite capable of single alternation but do not corroborate Hunter's assertion that rats are unable to learn a double-alternation task. Rather, these data support the more recent findings of other investigators who have observed at least some degree of successful double-alternation responding in the rat (e.g., Heise et al., 1969; Khavari, 1970; Schlosberg \& Katz, 1943).

An attempt to reinforce double alternation on an FR 2 schedule was totally unsuccessful. The apparent inability of the rat to learn an extended series of responses (e.g., LLRRLLRR) corroborated Hunter and Nagge's (1931) findings concerning the total lack of learning shown by rats in the "temporal maze" when extended response sequences were required.

Notwithstanding the rats' failure to maintain accurate doublealternation responding on an FR 2 schedule of reinforcement, the present experiment demonstrated that rats are capable of learning an uncued free operant double-alternation task. However, learning was obviously difficult and was not as extensive as learning of a single-alternation task. Although the discrepancy in performance between single alternation and double alternation may have been attributable to some genetic limitation in the rats' ability to learn double-alternation sequences, the possibility remains that initial training on the single-alternation task interfered with subsequent learning of the double-alternation sequence. While the present data do not permit any direct assessment of this potential source of negative transfer, learning of the double-alternation sequence might, in fact, be enhanced if training began with a double-alternation task.

\section{REFERENCES}

Bloom, J. M., \& CAPaldi, E. J. The behavior of rats in relation to complex patterns of partial reinforcement. Journal of Comparative and Physiological Psychology, 1961, 54, 261-265.

Ferster, C. B. Intermittent reinforcement of a complex response in a chimpanzee. Journal of the Experimental Analysis of Behavior, 1958, 1, 163-165.

Ferster, C. B. Intermittent reinforcement of matching to sample in the pigeon. Journal of the Experimental Analysis of Behavior, 1960, 3, 259-272.

Ferster, C. B., \& Skinner, B. F. Schedules of reinforcement. New York: Appleton-Century-Crofts, 1957.

Heise, G. A., Keller, C., Khavari, K. A., \& Laughlin, N. Discrete trial alternation in the rat. Journal of the Experimental Analysis of Behavior, 1969, 12, 609-627.

HunTer, W. S. The temporal maze and kinaesthetic sensory processes in the white rat. Psychobiology, 1920, 2, 1-17.

Hunter, W. S., \& Nagge, J. W. The white rat and the double alternation temporal maze. Journal of Genetic Psychology, 1931, 39, 303-319.

Kelleher, R. T. Chaining and conditioned reinforcement. In W. K. Honig (Ed.), Operant behavior: Areas of research and application. New York: Appleton-Century-Crofts, 1966.

KhAVARI, K. A. Spacial single and double alternation learning in discrete trial operant situation. Journal of Genetic Psychology, 1970, 116, 241-246.

Livesey, P. J. Comparisons of double alternation performance of white rats, rabbits, and cats. Journal of Comparative and Physiological Psychology, 1965, 59, 155-158.

Ludvigson, H. W., \& Sytsma, D. The sweet smell of success: Apparent double alternation in the rat. Psychonomic Science, 1967, 9, 283-284.

Schlosberg, H., \& KATz, A. Double alternation lever-pressing in the white rat. American Journal of Psychology, 1943, 56, 274-282.

(Received for publication February 20, 1982.) 